\title{
Lipid-modifying therapy in the elderly
}

This article was published in the following Dove Press journal:

Vascular Health and Risk Management

14 May 2015

Number of times this article has been viewed

\author{
Ian Hamilton-Craig ${ }^{1,2}$ \\ David Colquhoun ${ }^{3,4}$ \\ Karam Kostner ${ }^{4,5}$ \\ Stan Woodhouse ${ }^{4,6}$ \\ Michael d'Emden ${ }^{4,7}$ \\ 'Griffith University School of \\ Medicine, Griffith Health Institute, \\ Gold Coast, QLD, Australia; ${ }^{2}$ Flinders \\ University School of Medicine, \\ Adelaide, SA, Australia, ${ }^{3}$ Wesley \\ Medical Centre, Auchenflower, \\ QLD, Australia; ${ }^{4}$ University of \\ Queensland, Brisbane, QLD, Australia; \\ ${ }^{5}$ Department of Cardiology, Mater \\ Hospital, Woolloongabba, QLD, \\ Australia; ${ }^{6}$ Taylor Medical Centre, \\ Woolloongabba, QLD, Australia; \\ ${ }^{7}$ Department of Endocrinology, Royal \\ Brisbane Hospital, Herston, QLD, \\ Australia
}

\begin{abstract}
Cardiovascular disease (CVD) mortality and morbidity increases with increasing age, largely as a result of increased lifetime exposure as well as increased prevalence of CVD risk factors. Hospitalization for CVD increases by a factor of over $18 \times$ for those aged $85+$ years versus those aged $<30$ years. In spite of this, life expectancy continues to increase, and in Australia for people reaching the age of 65 years, it is now 84 years in men and 87 years in women. The number of people for whom lipid management is potentially indicated therefore increases with aging. This is especially the case for secondary prevention and for people aged 65-75 years for whom there is also evidence of benefit from primary prevention. Many people in this age group are not treated with lipid-lowering drugs, however. Even those with CVD may be suboptimally treated, with one study showing treatment rates to fall from $\sim 60 \%$ in those aged $<50$ years to $<15 \%$ for those aged $85+$ years. Treatment of the most elderly patient groups remains controversial partly from the lack of randomized trial intervention data and partly from the potential for adverse effects of lipid therapy. There are many complex issues involved in the decision to introduce effective lipid-lowering therapy and, unfortunately, in many instances there is not adequate data to make evidence-based decisions regarding management. This review summarizes the current state of knowledge of the management of lipid disorders in the elderly and proposes guidelines for management.
\end{abstract}

Keywords: lipid management, elderly, statins

\section{Introduction}

The average life expectancy of people in Western cultures has increased significantly over the last 50 years with the average age of death for a person reaching the age of 65 now being 85 years for men and 87 years for women. Persons living past the age of 65 (the elderly) can now expect to live for nearly a further 19-22 years. Importantly, cardiovascular disease (CVD) accounts for $>75 \%$ of deaths in the elderly. Effective lipid management in the elderly should reduce the risk of developing CVD with improvement of quality of life and potentially a further increase in longevity. There are many complex issues involved in the decision to introduce effective lipid-lowering therapy, but, unfortunately, in many instances there is not adequate data to make evidence-based decisions regarding management. In this paper, we review the current state of knowledge of the management of lipid disorders in the elderly.

\section{Current suboptimal management of the elderly}

Management of hyperlipidemia in the elderly may be suboptimal for several reasons:

1. The statistical relationship between blood cholesterol and CVD is less strong in the elderly compared to the middle-aged. ${ }^{1}$ 
2. Physician uncertainty exists with regard to the benefits of lipid management.

3. There are concerns about adverse drug reactions (ADRs) including increased risk of statin-associated myalgia and other statin-related ADRs. ${ }^{2,3}$

4. CVD risk calculators are unreliable in elderly patients, in whom physicians may either underestimate or overestimate CVD risk. ${ }^{4}$

In view of these factors, the elderly are treated less frequently with lipid-lowering therapy. For example, Massing et al analyzed 56,320 medical records from 296 medical practices in the US, and showed that lipid medication rates in patients with coronary artery disease (CAD) declined with increasing age from $\sim 60 \%$ in those aged 50 years to $<15 \%$ in those aged $\geq 85$ years. ${ }^{5}$ Dose titration of statins was inversely related to age; doses above the starting dose were given to $60 \%$ of patients aged $21-44$ years and to $35 \%$ of patients aged $\geq 85$ years. Rates of prescribing non-lipid medications were similar at all ages, however. Figure 1 shows the mean odds ratios (ORs) for treatment with lipid therapy according to age. ${ }^{5}$ The ratio declined with advancing age and was lowest in the age group $>85$ years.

More recent studies have also shown significant lipid treatment gaps in primary care, as in the AusHEART study in middle-aged patients aged $\geq 55$ years in which patients aged $>75$ years were excluded. ${ }^{6}$

Simons and Chung used records of the Pharmaceutical Benefits Scheme between 2006 and 2013 in 276,212 patients at high CVD risk and mean age 66 years. Of this group, $42 \%$ had not received lipid-lowering drugs during the study period. ${ }^{7}$ There was a U-shaped relationship with age, with

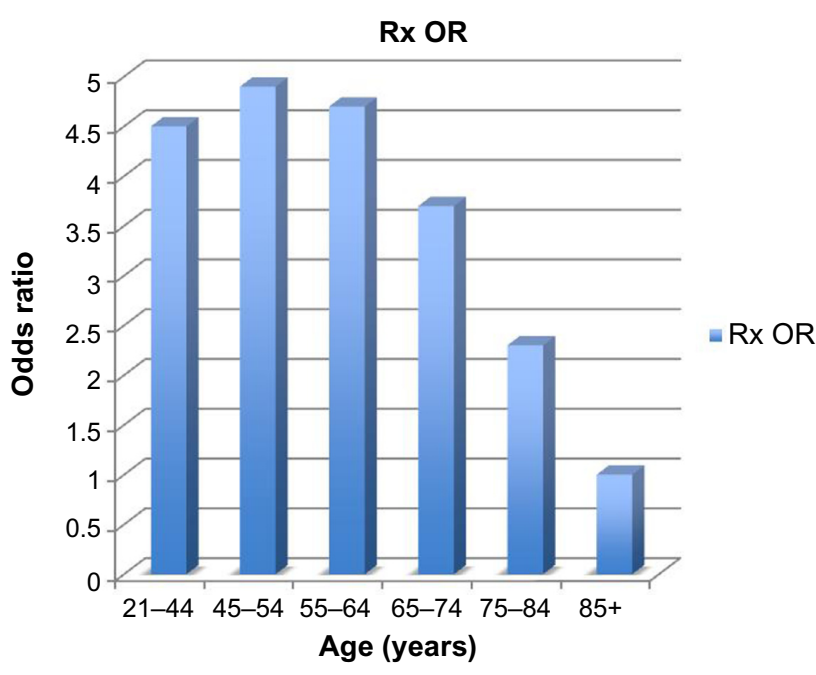

Figure I Mean odds ratios for treatment with lipid therapy according to age. the proportions not receiving drugs being highest in those aged $<41$ years and $>81$ years. $^{?}$

We previously observed in a survey of general practice in Australia that about $50 \%$ of patients with severe hypercholesterolemia (total cholesterol $[\mathrm{TC}]>7.5 \mathrm{mmol} / \mathrm{L}$ ) were not treated with lipid-lowering therapy. ${ }^{8}$ This proportion was similar for all age groups, and it appeared (at least for severe hypercholesterolemia) that physician inertia may not only be confined to the elderly but may apply to other age groups as well. ${ }^{8}$

\section{Appropriateness of therapy}

Holmes et al have proposed a process for medication prescribing in patients late in life that builds on the principles of appropriate prescribing and includes consideration of the remaining life expectancy, goals of care, quality of life, and potential benefits of medications (Table 1). ${ }^{9}$

In their model, four steps in medication decision making form a pyramid, visually representing the appropriate medications at any level. The model identifies patients for whom statin therapy is the least appropriate (limited life expectancy, palliative goals of care, treatment targets focused on symptom management) versus patients most appropriate for statin therapy (longer life expectancy, longer time until benefit, more aggressive goals of care focused on preventive strategies). ${ }^{9}$

\section{Life expectancy}

Life expectancy in most countries including Australia is increasing progressively. For those aged 65 years, life expectancy is a further 19 years and 22 years - to 84 years in men and 87 years in women. ${ }^{10}$ For men and women aged 85 years, further life expectancy is 5.9 years and 7.1 years, respectively. ${ }^{11}$

Table I The Medication Appropriateness Index 9

I. Is there an indication for the drug?

2. Is the medication effective for the condition?

3. Is the dosage correct?

4. Are the directions correct?

5. Are the directions practical?

6. Are there clinically significant drug-drug interactions?

7. Are there clinically significant drug-disease/condition interactions?

8. Is there unnecessary duplication with other drugs?

9. Is the duration of therapy unacceptable?

10. Is this drug the least expensive alternative compared with others of equal usefulness?

Note: Reproduced with permission from Holmes HM, Hayley DC, Alexander GC, Sachs GA. Reconsidering medication appropriateness for patients late in life. Arch Intern Med. 2006;166:605-609. Copyright (c) (2006) American Medical Association. All rights reserved. ${ }^{9}$ 
There are, however, no reliable tools for predicting life expectancy in elderly Australians, other than through extrapolation of parameters known to affect survival such as CVD risk factors, family history, and other intuitive variables. Attempts to develop prognostic indices in older adults have met with varied success, and few indices have been independently validated. ${ }^{4}$ Use of the Lloyd-Jones algorithm for predicting CVD risk up to age 80 years is recommended by the International Atherosclerosis Society (IAS). ${ }^{12}$

Recent reviews have suggested the need for contemporary and comprehensive health care guidelines in the elderly. ${ }^{13,14}$ In this paper, we review the evidence for lipid therapy in the elderly and propose guidelines for therapy.

\section{Cardiovascular risk in the elderly}

Epidemiological studies have consistently shown that CVD risk increases with age in both sexes, and that increasing age is one of the most powerful factors predicting CVD risk. CVD in older patients is also associated with worse outcomes.

Figure 2 shows the CVD risk in the Australian population according to age and sex. ${ }^{15}$ Those aged 75 years or more have several-fold higher rates of CVD than those aged $<50$ years, the relationship between age and CVD risk being semiexponential.

\section{CVD risk factors in the elderly}

With increasing age, the burden of CVD risk factors (other than cigarette smoking, TC, and body mass index [BMI]) increases progressively, as shown in Table $2 .{ }^{16}$

In the elderly, gradients in CVD risk depend on the number of risk factors present, as in younger age groups. Data from the Dubbo study are shown in Figure 3. ${ }^{1}$

The Prospective Studies Collaboration showed that the strength of the relationship between usual cholesterol levels and vascular mortality was inversely related to age (Figure 4). ${ }^{17}$

These data show a much steeper gradient between the hazard ratio (HR) for vascular mortality in younger compared to older patients.

In Figure 4, the HR increased from $\sim 0.8$ (with TC $4.5 \mathrm{mmol} / \mathrm{L}$ ) to $\sim 12$ (with TC $8.0 \mathrm{mmol} / \mathrm{L}$ ) in patients aged $40-49$ years, while in patients aged $80-89$ years the HR rose from $\sim 128$ to 256 for the same TC levels.

Figure 4 also suggests that lowering cholesterol in younger patients will result in greater relative risk reduction (RRR, by a factor of $\sim 15$ ) compared to the oldest group (by a factor of $\sim 2$ ). Absolute risk reduction, however, is greater in older patients, with HR falling by $\sim 128$ in patients in the oldest age group and by $\sim 11$ in the youngest.

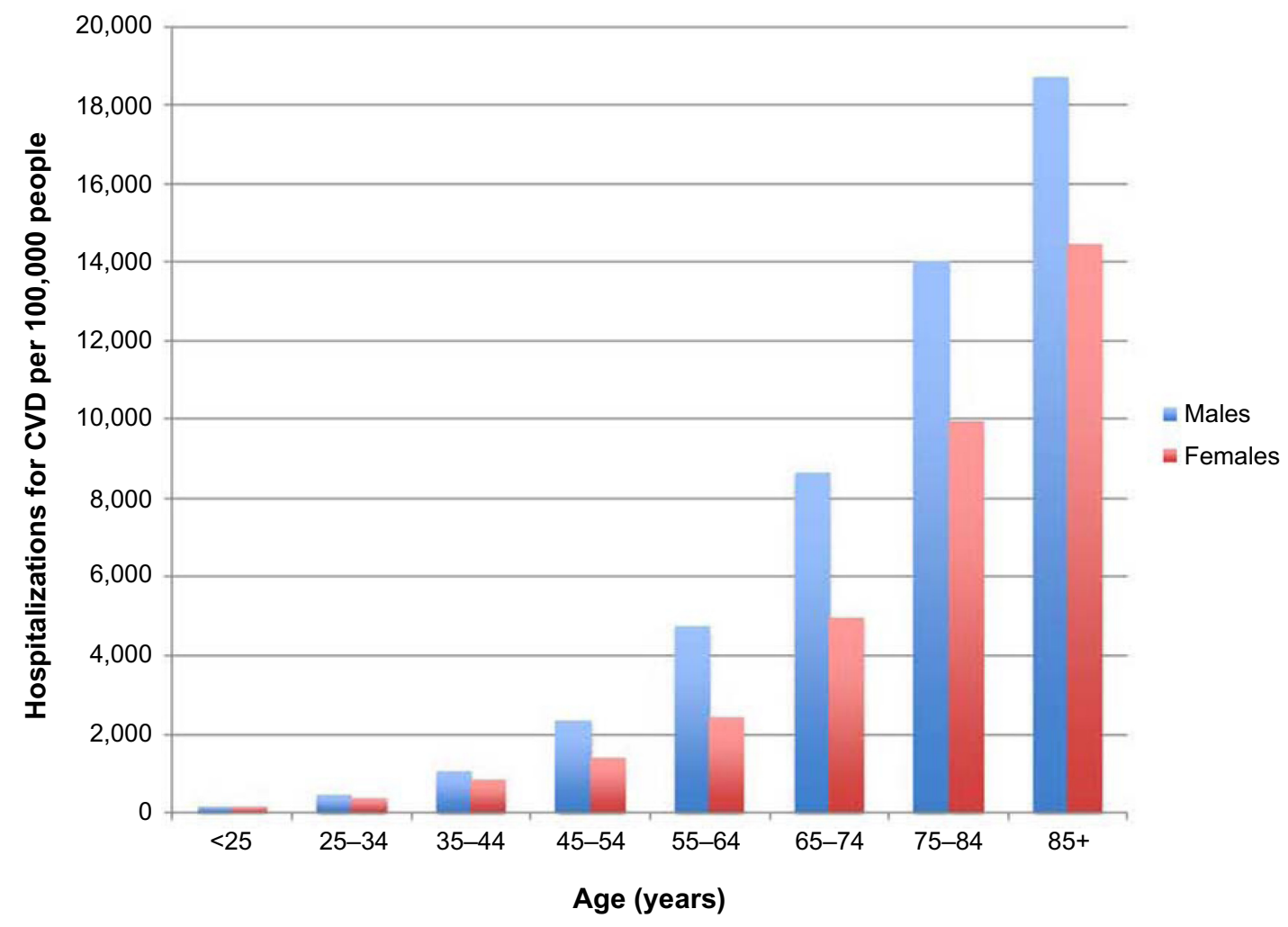

Figure 2 Hospitalizations for CVD per 100,000 people in the Australian population according to age and sex. 
Table 2 Prevalence of CVD risk factors (\%) by age and sex in the Framingham Study ${ }^{16}$

\begin{tabular}{|c|c|c|c|c|c|c|}
\hline Age & ECG-LVH ${ }^{a}$ & ECG-LVH & $\mathrm{DM}^{\mathrm{b}}$ & DM & $H^{c}{ }^{c}$ & $\mathrm{HT}$ \\
\hline & $M$ & $\mathbf{F}$ & $M$ & $\mathbf{F}$ & $M$ & $\mathbf{F}$ \\
\hline $55-64$ & 2.7 & 1.7 & 5.5 & 4.2 & 26.8 & 31.5 \\
\hline $65-74$ & 3.6 & 3.2 & 10.9 & 7.2 & 38.0 & 47.6 \\
\hline $75-84$ & 4.2 & 4.9 & 13.2 & 10.2 & 48.4 & 59.9 \\
\hline $85-94$ & 5.9 & 9.4 & 14.2 & II.I & 47.8 & 65.6 \\
\hline
\end{tabular}

Notes: ${ }^{2}$ Electrocardiographic evidence of left ventricular hypertrophy; 'diabetes mellitus (treated for diabetes or blood glucose $\geq 200 \mathrm{mg} / \mathrm{dL}$ ); chypertension ( $\geq 160 / 95$ ). Coronary heart disease risk factors in the elderly. Kannel WB. () 2002 The American Journal of Geriatric Cardiology. Reproduced with permission of Blackwell Publishing Ltd. ${ }^{16}$ Abbreviations: CVD, cardiovascular disease; ECG, electrocardiographic evidence; LVF, left ventricular hypertrophy; DM, diabetes mellitus; $\mathrm{HT}$, hypertension; M, male; F, female.

The "elderly paradox" is based on epidemiological studies and refers to the absolute benefit being greater in the elderly even though the risk attributable to cholesterol is much less. The number of patients needed to be treated to prevent a coronary event is therefore less in the elderly due to a higher absolute risk. There is potentially greater

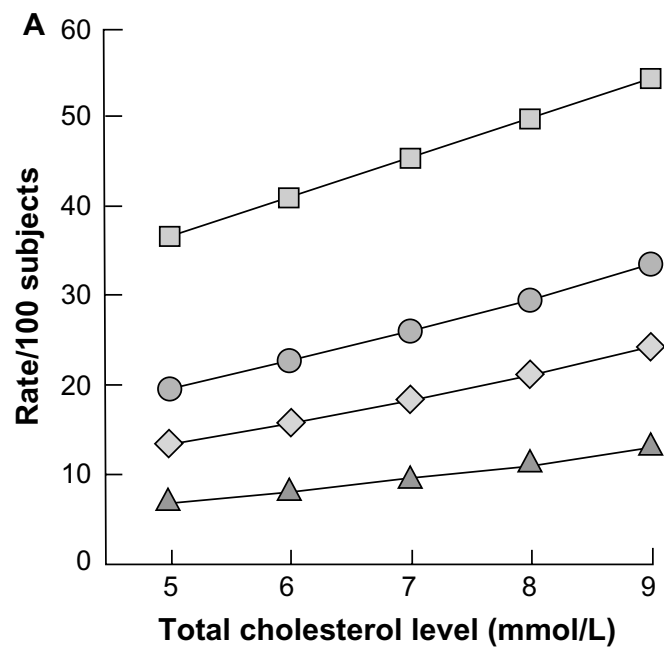

$\triangle$ No other risk factors $\quad-\mathrm{O}+$ + Smoking $\checkmark$ Antihypertensive medication $-\square-+$ Diabetes

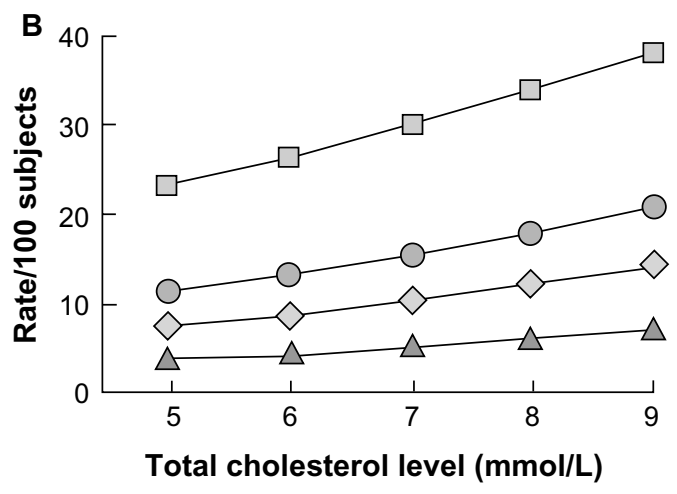

Figure 3 Cardiovascular risk according to risk factors and total cholesterol levels in the Dubbo study.

Notes: Reference group: not taking antihypertensive medication; systolic pressure $140 \mathrm{mmHg}$; non-smoking; high-density lipoprotein level $1.10 \mathrm{mmol} / \mathrm{L}$; no diabetes.

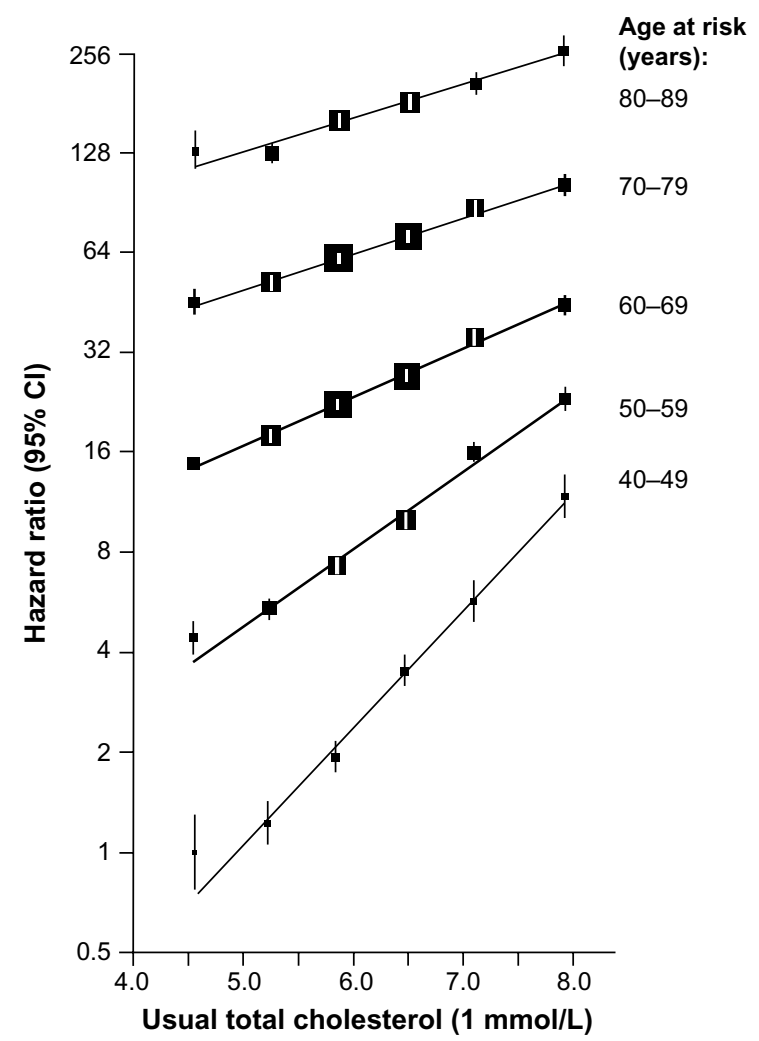

Figure 4 Vascular mortality according to age and total cholesterol level in the Prospective Studies Collaboration.

harm from drug therapy in the elderly and potentially less benefit due to other illnesses that impact on the quality of life and survival. Obviously, the very elderly have reduced life expectancy compared to middle-aged patients. As a result of these general considerations, the elderly may be denied lipid treatments that could have a significant positive effect on morbidity and the associated quality of life.

\section{Measurement of lipids in the elderly}

The proportion of private lipoprotein tests in Queensland in 1 year increases linearly with increasing age (Figure 5 ). ${ }^{8}$ The lowest proportion of lipoprotein tests occurs under age 25 years $(\sim 12 \%)$, compared with $\sim 50 \%$ for age $45-54$ years, $>80 \%$ for age $65-74$ years, and $92 \%$ for age $>74$ years. Measurement of lipids would not seem to be a limiting step to treatment in older patients.

\section{Estimating CVD risk in the elderly}

The IAS recently recommended use of lifetime (long-term) risk prediction from age 50 years to 80 years for clinical intervention on low-density lipoprotein cholesterol (LDL-C) and atherogenic lipoproteins (Table 3). ${ }^{18}$ The exclusion of patients older than 80 years reflects the lack of data in this age group. 


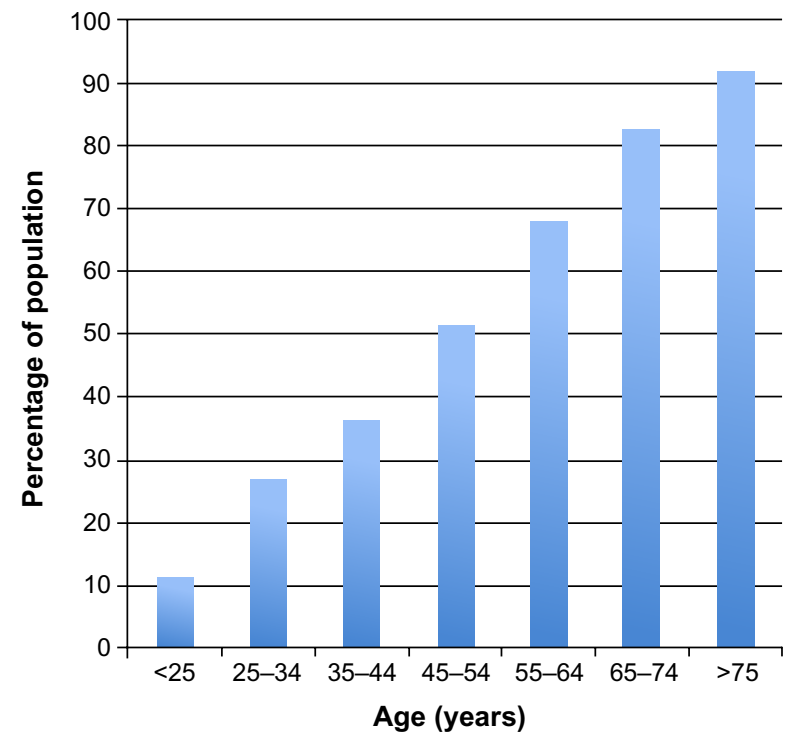

Figure 5 Proportion of private lipoprotein tests in Queensland in I year according to age.

For primary prevention, risk to age 80 for atherosclerotic CVD can be stratified into high $(>45 \%)$, moderately high $(30 \%$ $44 \%)$, moderate $(15 \%-30 \%)$, and low $(<15 \%)$ (Table 3$)$.

According to the IAS, QRISK (a prediction algorithm for CVD) appears to be reliable for Western Europe and is ethnicity specific (for the UK). ${ }^{19}$ For world populations, the IAS recommends the Lloyd-Jones/Framingham algorithm for estimating absolute risk for total atherosclerotic CVD to age $80 .{ }^{20}$ Calculated risk can be recalibrated based on the coefficients determined by national comparisons with Framingham estimates. If recalibration values were unavailable, treatment can be focused on individual risk factors.

\section{Coronary calcium score}

In the elderly, risk profiling using variations of the Framingham equations overestimate risk due to the weighting of age in the calculation. Many clinicians do not use risk calculators and believe that the impression of risk is as accurate, and to some extent this is correct. ${ }^{21}$

Table 3 Long-term risk for atherosclerotic cardiovascular disease by age 80 (from age 50 )

\begin{tabular}{ll}
\hline Long-term risk category & $\begin{array}{l}\text { Absolute risk for atherosclerotic } \\
\text { cardiovascular disease (\%) }\end{array}$ \\
\hline Low & $<15$ \\
Moderate & $15-30$ \\
Moderately high & $30-44$ \\
High & $>45$ \\
\hline
\end{tabular}

Note: Coronary heart disease risk factors in the elderly. Kannel WB. (C) 2002 The American Journal of Geriatric Cardiology. Reproduced with permission of Blackwell Publishing Ltd. ${ }^{16}$
Coronary calcium scoring has emerged as an independent predictor of CAD and it may be more useful in the elderly in terms of predicting risk. Recently, a review of coronary calcium scoring in asymptomatic individuals assessing mortality in patients older than 75 years has been published. ${ }^{22}$ A zero coronary artery calcium (CAC) score is associated with a 5.6year survival of $98 \%$, similar to those in other groups with a cardiac calcium score of zero, which is approximately $99 \%$. The study followed up 44,052 asymptomatic individuals in North America. The increasing CAC independently predicted an increased risk of all-cause mortality in all age groups. In the $>75$ age group, a CAC of $>400$ was associated with $16 \times$ greater mortality than a CAC equaling zero. In this study, CAC also predicted all-cause mortality in those aged $<45$ years. As this test is such a powerful predictor, many patients who would otherwise not be on therapy would be commenced on therapy to lower the CVD risk, and a significant number of elderly would not need to go on to drug therapy.

\section{Lipid management in the elderly: evidence for benefit from statin trials}

Roberts et al showed reduced total mortality (15\% reduction), coronary heart disease (CHD) mortality (23\% reduction), fatal or nonfatal stroke (24\% reduction), and fatal or nonfatal myocardial infarction (MI) (26\% reduction) in a meta-analysis of over 50,000 patients aged $>60$ years treated with statins (Figure 6). ${ }^{23}$ They concluded, "statin therapy should be offered to older patients at high risk", and "statin treatment is associated with clearly reduced mortality risk of cardiovascular patients aged 75 years or older".

Figure 7 shows the number of major CVD events avoided with statin therapy for all age groups, according to baseline 5-year risk of a CVD event in the meta-analysis of the Cholesterol Treatment Trialists' Collaboration (CTTC). ${ }^{24}$

Figure 8 shows the effects on major vascular events per $1.0 \mathrm{mmol} / \mathrm{L}$ reduction in LDL-C at different levels of risk, by baseline age, according to the CTTC meta-analysis. ${ }^{24}$ The event rate increased with increasing age from $\leq 60$ years to $>70$ years, while the RRR was of a similar order of magnitude for all age groups. ${ }^{24}$ For every mmol/L reduction in LDL-C achieved with statin treatment, the relative risk reductions in major vascular events for the age groups $<=60$ years, $>60$ to $<=70$ years and $>70$ years were $0.77,0.78$ and 0.83 , respectively (all $P<0.001$ ).

The CTTC data strongly support use of statins in the elderly for CVD risk reduction. ${ }^{24}$

One trial (PROSPER) was designed to test the benefits of statin therapy specifically in the elderly. ${ }^{25}$ In 5,804 men 


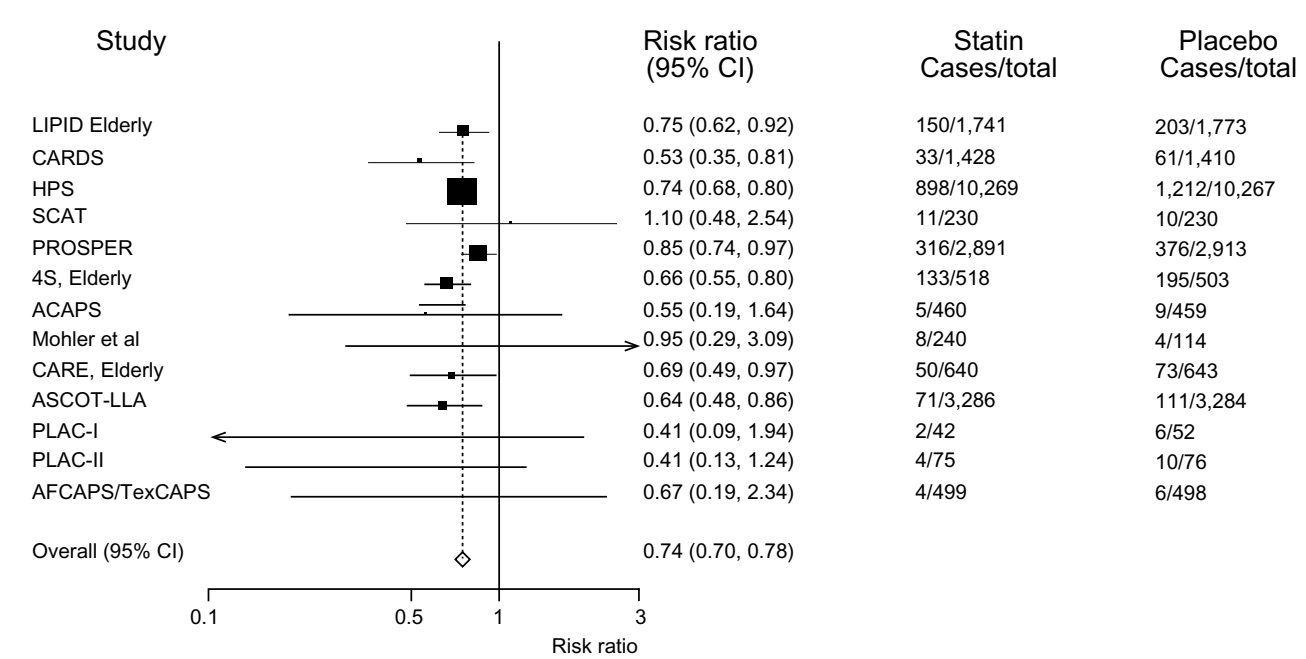

Figure 6 Meta-analysis of patients with cardiovascular disease aged $>60$ years according to statin versus placebo treatment.

Note: Reproduced from Roberts CG, Guallar E, Rodriguez A. Efficacy and safety of statin monotherapy in older adults: a meta-analysis. J Gerontol A Biol Sci Med Sci. 2007;62:879-887, by permission of Oxford University Press. ${ }^{23}$

Abbreviations: LIPID, the Long-Term Intervention with Pravastatin in Ischaemic Disease (LIPID) Study Group; CARDS, Collaborative Atorvastatin Diabetes Study; HPS, Heart Protection Study; SCAT, Simvastatin/Enalapril Coronary Atherosclerosis Trial; PROSPER, Pravastatin in elderly individuals at risk of vascular disease; 4S, Scandinavian Simvastatin Survival Study; ACAPS, Asymptomatic Carotid Artery Progression Study; CARE, the Cholesterol and Recurrent Events trial; ASCOT-LLA, Anglo-Scandinavian Cardiac Outcomes Trial--Lipid Lowering Arm (ASCOT-LLA); PLAC-I, Pravastatin Limitation of Atherosclerosis in the Coronary arteries I; PLAC-II, Pravastatin Limitation of Atherosclerosis in the Coronary arteries II; AFCAPS/TexCAPS, Air Force/Texas Coronary Atherosclerosis Prevention Study.

and women aged 70-82 years (2,520 Scottish subjects), a significant benefit occurred with pravastatin therapy compared with placebo. There was $19 \%$ reduction in coronary events after a mean of 3.2 years $(P=0.006)$, with no impact on stroke or total mortality and a suggestion of increased cancer risk. ${ }^{25}$ The recently published long-term follow-up of the PROSPER study over 8.6 years showed $20 \%$ reduction in coronary events with no effect on cancer, stroke, or total mortality. ${ }^{25}$ It was

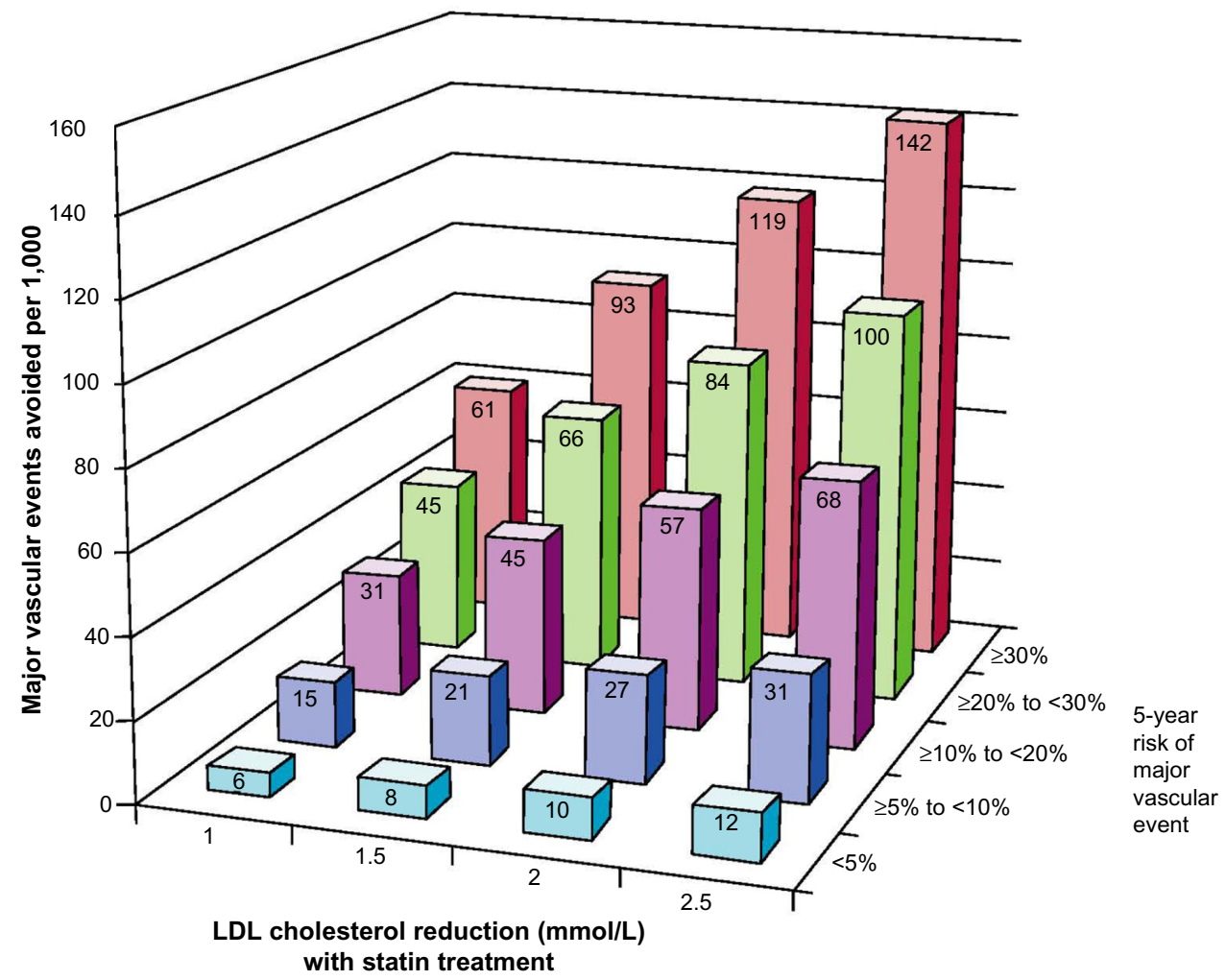

Figure 7 Predicted 5-year benefits of LDL cholesterol reductions with statin treatment at different levels of risk in Cholesterol Treatment Trialists' Collaboration metaanalysis. 


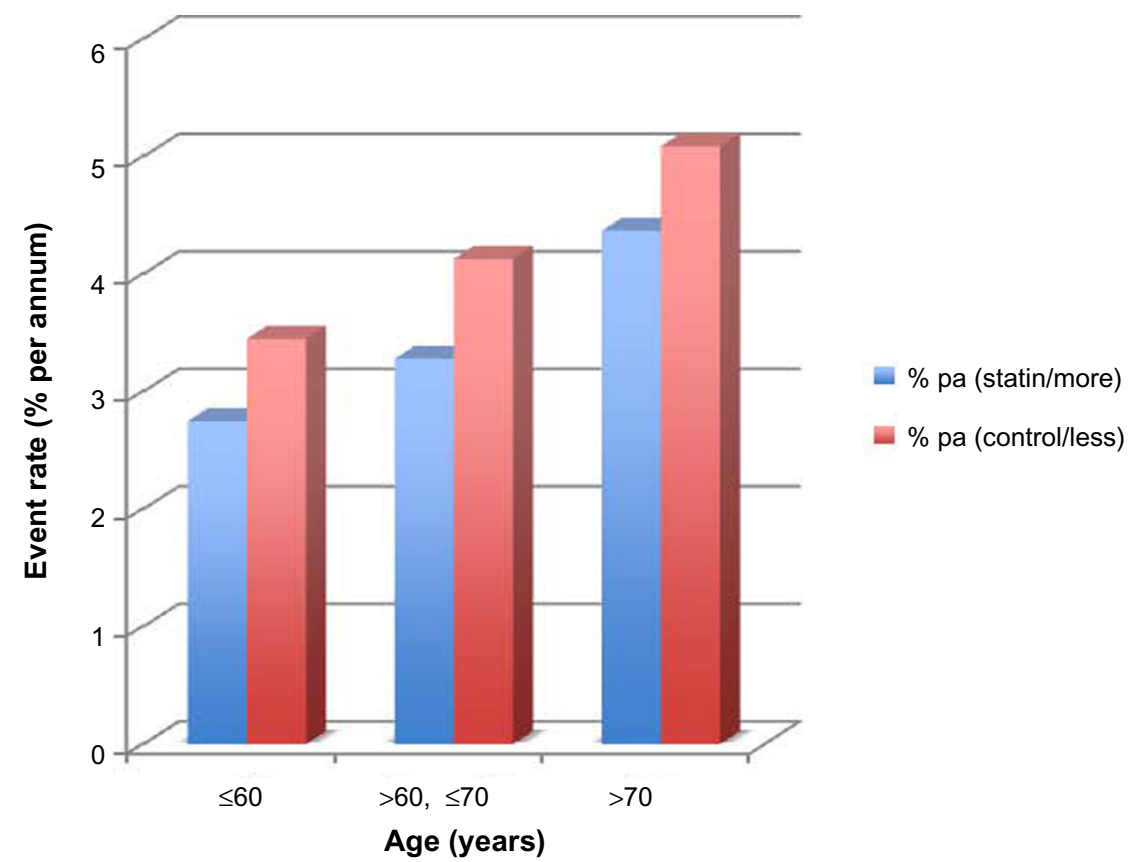

Figure 8 Major vascular event rate with statin treatment according to age in Cholesterol Treatment Trialists' Collaboration meta-analysis.

suggested that the lack of benefit for survival possibly reflected competing mortality from other causes.

Figure 9 shows the reduction in coronary death or hospitalization for MI with pravastatin therapy in the Scottish cohort of the PROSPER study. ${ }^{25}$
Other data showing benefit from statin therapy in elderly patients are shown in Figures 10 and 11. ${ }^{26,27}$ Allen Maycock et al showed that statin therapy was associated with reduced mortality across all age groups of individuals with significant coronary disease, including very elderly patients (Figure 10). ${ }^{26}$

\section{Coronary death or hospitalisation for MI (Scottish cohort)}

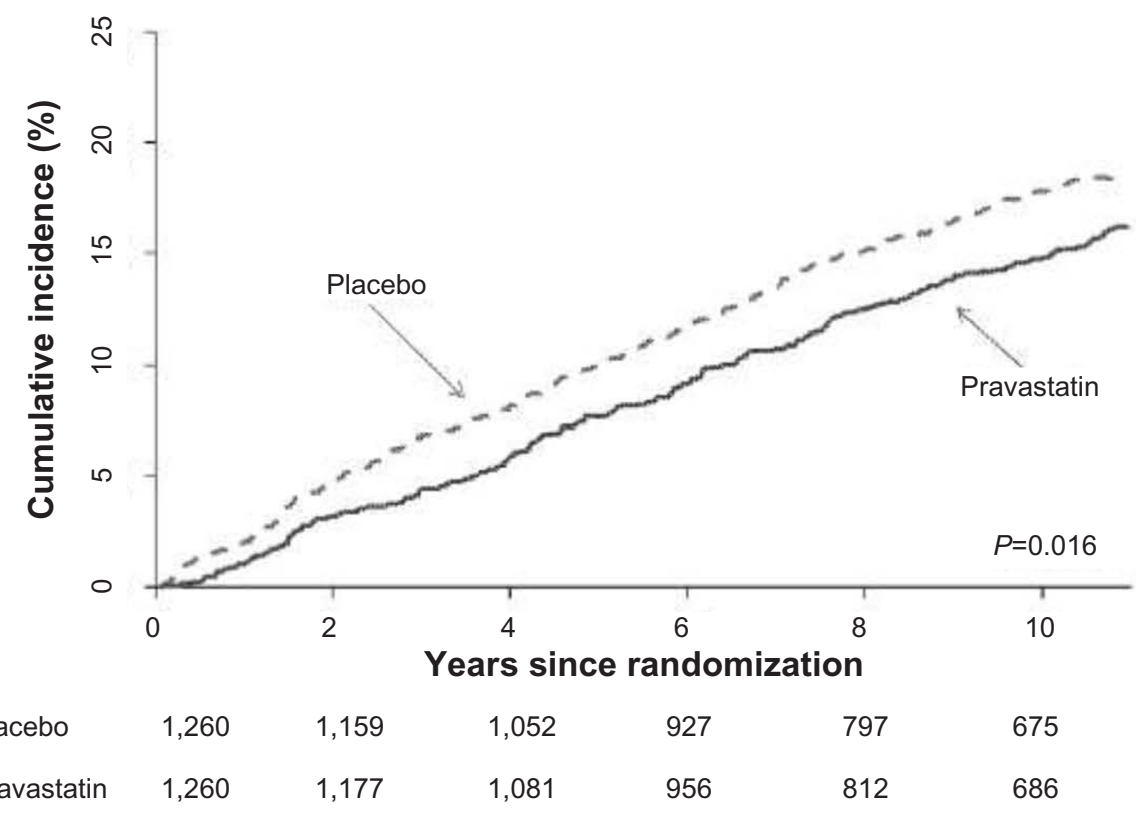

Figure 9 Reduction in coronary death or hospitalization for myocardial infarction with pravastatin versus placebo treatment.

Notes: Numbers of patients included in the study are provided according to year since randomization for patients treated with either placebo (upper line of $X$ axis) or pravastatin (lower line of X axis). ( 2013 Lloyd SM, Stott DJ, de Craen AJ, et al. Long-term effects of statin treatment in elderly people: extended follow-up of the PROspective Study of Pravastatin in the Elderly at Risk (PROSPER). PLoS One. 2013;8:e72642. ${ }^{25}$

Abbreviation: Ml, myocardial infarction. 


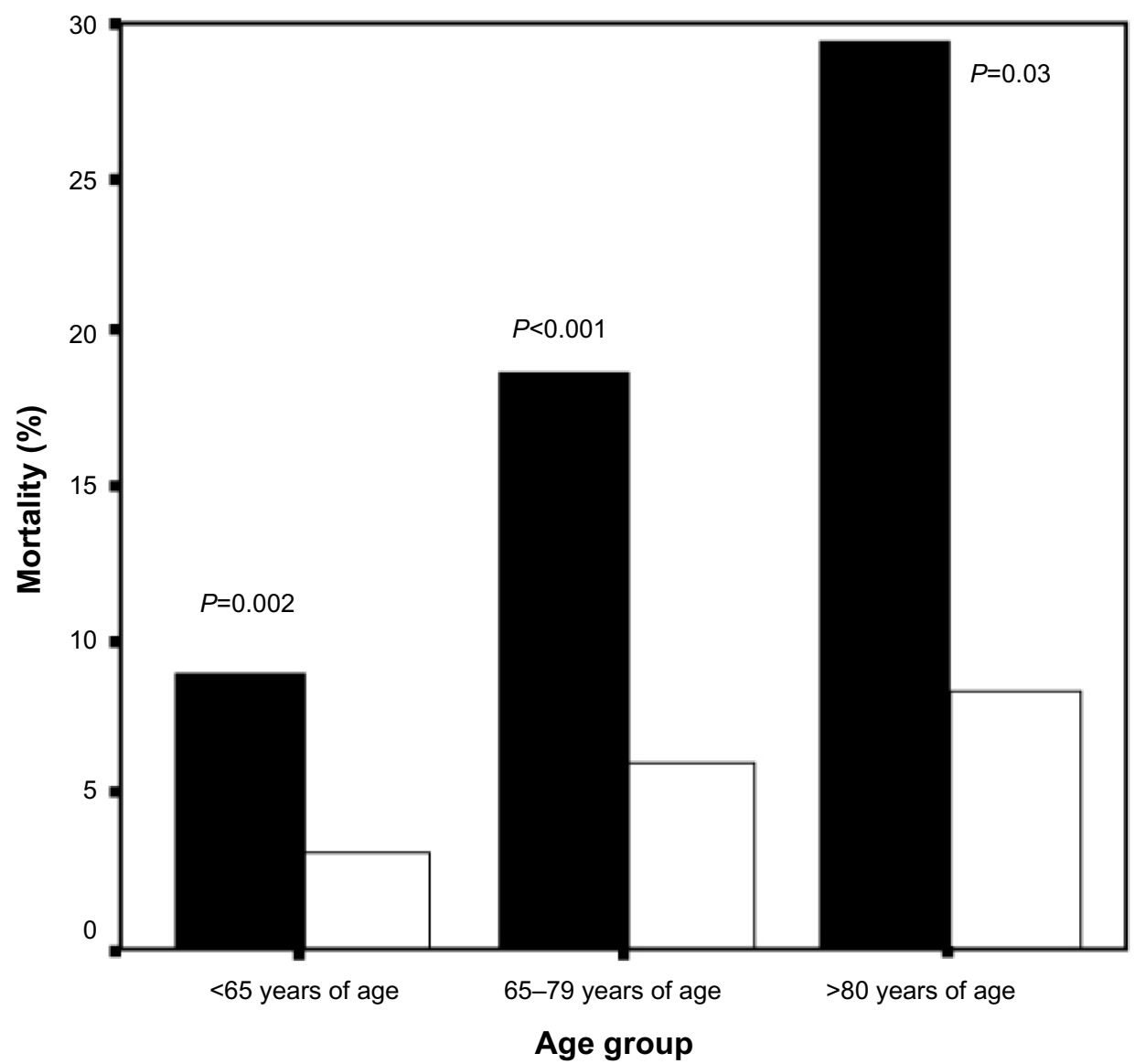

Figure 10 Mortality reduction in cardiovascular disease patients with statin treatment according to age. Note: Black bars: no statin treatment; white bars: statin treatment.

Miettinen et al investigated the benefits of simvastatin therapy compared with placebo in elderly subjects with CHD of the Scandinavian Simvastatin (4S) Study (Figure 11). ${ }^{27}$ The cohort of 4,444 subjects included 1,021 patients aged $\geq 65$ years, with 5.4 mean years of follow-up. In the

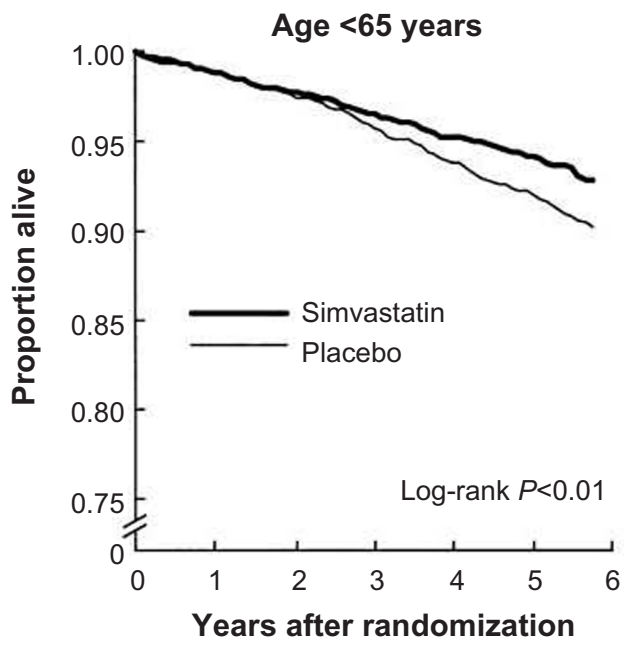

elderly cohort, there were significantly lower rates of total mortality (34\%), CHD mortality (43\%), major CHD events (34\%), and revascularizations (41\%).

In the LIPID study of 9,014 subjects, 3,514 were aged 65-75 years. Patients had previous MI or unstable angina

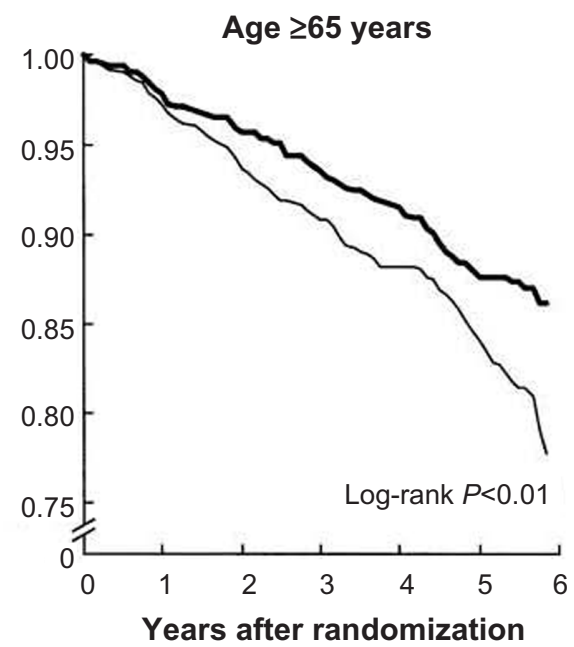

Figure I I Benefits of simvastatin versus placebo treatment in Scandinavian Simvastatin Survival Study. Note: The $Y$ axis of both graphs refers to proportion alive where $1.00=100 \%$. 
and were treated with pravastatin or placebo for 6 years. ${ }^{28}$ Similar RRR was observed with pravastatin therapy for older (65-75 years) and younger ( $<65$ years) age groups. For every 1,000 older patients treated, pravastatin prevented 45 deaths, 33 MIs, 32 unstable angina events, 34 revascularizations, 13 strokes, and 133 major CVD events compared with 22 deaths and 107 major CVD events in younger patients. ${ }^{28}$ It was concluded that the absolute benefit of treatment is significantly greater in older patients with previous CHD because they are at greater risk.

In a substudy of the Cholesterol and Recurrent Events (CARE) trial, Lewis et al investigated the effect of pravastatin on cardiovascular events in older patients aged $\geq 65$ years with previous MI and cholesterol levels $<6.2 \mathrm{mmol} / \mathrm{L}(240 \mathrm{mg} / \mathrm{dL})$ and LDL-C levels 3.0-4.5 mmol/L (115-174 mg/dL) ${ }^{29}$ Over 5 years in 1,283 subjects, pravastatin therapy compared with placebo reduced CHD and stroke by $32 \%$ and $40 \%(P<0.001$ and $P=0.03$ ), respectively. For every 1,000 patients treated, pravastatin prevented 225 hospitalizations in older patients compared to 121 in younger patients. ${ }^{29}$

\section{Adherence in the elderly}

Adherence is the key to obtaining benefit of proven drug therapy. Age is a factor, and within the elderly age bracket, probably the "very old" have even poorer long-term persistence with therapy. Webster packs and similar aids by pharmacies have been shown to improve adherence.

In a recent study of 259 patients discharged after an acute MI, persistence was assessed. ${ }^{30}$ At discharge, 99.2\% were prescribed antiplatelets, $86.5 \%$ betablockers, 95\% statins, and 90.4\% angiotensin-converting enzyme (ACE) inhibitors. Persistence at 1 year post discharge was worse in those $>75$ years, dropping from $74.1 \%$ for the combination of all drug classes to $37.8 \% .^{30}$

\section{Triglycerides in the elderly}

In a study of triglycerides (TGs) as risk factors for CHD, the adjusted ORs of association were 1.57 (95\% CI, 1.10-2.24) and 1.76 (CI, 1.39-2.21) for the EPIC-Norfolk and Reykjavik studies, respectively, in which the mean ages were 74 years and 70 years, respectively. ${ }^{31}$ Meta-analyses of these and other studies confirm that TGs are independent risk factors for CVD. ${ }^{31,32}$ Risk of CHD increases with fasting TGs above $1.7 \mathrm{mmol} / \mathrm{L}(\sim 150 \mathrm{mg} / \mathrm{dL})$. About one-third of adults have TGs in this range. ${ }^{33}$

No randomized trials of therapy with fibrates have been shown to benefit patients with elevated TG and low highdensity lipoprotein cholesterol (HDL-C) in elderly subjects.

However, recent meta-analyses of the five prospective, randomized, placebo-controlled trials demonstrate robust and consistent benefit for reducing CHD events in patients with fasting TCs $>2.3 \mathrm{mmol} / \mathrm{L}(\approx 200 \mathrm{mg} / \mathrm{dL}), \mathrm{OR}=0.65$ (0.54-0.78 [95\% CI]). ${ }^{34}$

The European Society of Cardiology/European Atherosclerosis Society (ESC/EAS) Guidelines recommend use of drugs to lower triglycerides if after lifestyle measures fasting triglycerides are $>2.3 \mathrm{mmol} / \mathrm{L}(\approx 200 \mathrm{mg} / \mathrm{dL}) .{ }^{35}$ Treatment is meant to be for patients deemed at "high total CV risk". High total CV risk is defined as either markedly elevated single risk factors such as familial dyslipidemia and severe hypertension, or a calculated SCORE $\geq 5 \%$ and $<10 \%$ for a 10 -year risk of fatal CVD. ${ }^{35}$ When fasting TGs are very high $>10 \mathrm{mmol} / \mathrm{L}(\approx 500 \mathrm{mg} / \mathrm{dL})$, risk of pancreatitis is clinically significant. The ESC/EAS guidelines note "actions to prevent acute pancreatitis are mandatory". Actions are to restrict fat $(10 \%-15 \%$ of total daily energy intake), abstinence of alcohol, fibrate therapy (fenofibrate), and n-3 fatty acids (2-4 g/day of eicosapentaenoic acid [EPA] and docosahexaenoic [DHA]) or nicotinic acid. ESC/EAS recommendations for drug treatment of elevated TGs are Class I/Level B for fibrates, Class IIa/Level B for n-3 fatty

Table 4 Guidelines on lipid management in the elderly by different groups: summary

Adult Treatment Panel III of National Cholesterol Education Program

American Society of Geriatric Cardiology National Institute for Health and Clinical Excellence

National Collaborating Centre for Primary Care

European Society of Cardiology/ European Atherosclerosis Society
I. Statins recommended for secondary prevention.

2. Clinical judgment used for primary prevention due to unreliable risk scoring assessments.

Treat individuals at high CVD risk who are otherwise healthy Clinical assessment used to guide therapy for whom appropriate risk calculator not available.

People aged 75 or over at increased CVD risk and likely to benefit from statins.

Treatment guided by benefits vs risk of treatment, informed preference, comorbidities that may make treatment inappropriate.

Very limited evidence for treatment above age 80-85 years. Clinical judgment should guide decisions in the very old.

Note: Reprinted from Heart Lung Circ 20I4;23. Noaman S, Ibrahim JE, Grenfell R Prescribing statins for cardiovascular disease prevention in the old: an absence of evidence and an absence of guidelines 619-624. Copyright (C20I4 with permission from Elsevier. ${ }^{14}$ 
acids, Class IIa/Level C for statin and fibrate, and Class IIb/ Level B for fibrate and n-3 fatty acids. ${ }^{35}$

\section{National and international guidelines for lipid management in the elderly}

Several recent guidelines on lipid management include sections on the elderly. They show some differences in approach but are generally consistent with each other (Table 4). ${ }^{14}$

\section{American College of Cardiologyl American Heart Association}

The American College of Cardiology/American Heart Association (ACC/AHA) 2013 guideline recommends the use of Pooled Cohort Equations for the estimation of the 10-year risk in those 76-79 years old to help guide treatment decision making. ${ }^{36}$ Evidence from randomized clinical trials supports continuing statin therapy beyond 75 years of age in patients already taking and tolerating them. Moderate-intensity statin use was supported for secondary prevention, but, for primary prevention in this age group, comorbidities, safety, and priorities of care should all be considered. Before initiating statins for primary prevention, therefore, discussion of CVD reduction benefits, risk of ADR, drug-drug interactions, and patient preference is recommended. ${ }^{36}$

\section{European Society of Cardiologyl European Atherosclerosis Society}

The ESC/EAS guidelines include a significant section on lipid management of the elderly. ${ }^{35}$ Summary statements were: "Risk reduction in individuals older than 65 years is essential because two-thirds to three-quarters of them have either clinical CAD or subclinical atherosclerotic disease. Almost 25\% of men and $42 \%$ of women older than 65 years have a TC level $>6 \mathrm{mmol} / \mathrm{L}$ ", and "According to published data, elderly individuals are a high risk group who could benefit significantly from lipid-lowering therapy to reduce $\mathrm{CV}$ morbidity and mortality. Evidence for treatment above the age of 80-85 years is very limited, and clinical judgment should guide decisions in the very old". 35

Primary prevention in the elderly was supported by the CTTC and other meta-analyses, in that RRR was similar in older and younger patients.

Because of the increased risk of drug ADR and drugdrug interactions, the ESC/EAS recommends that statins be started at low doses and then titrated to achieve optimal LDL-C levels.

Reduced compliance in the elderly was also discussed, which may result from perceived lack of benefit, drug ADR, cost, and CVD events occurring while on therapy. Improved explanation of benefits of treatment and treatment regimens may improve compliance.

\section{Australian National Vascular Disease Prevention Alliance}

The National Vascular Disease Prevention Alliance guidelines for the management of absolute risk in CVD 2012 recommends that patients with estimated 5-year CVD risk $\geq 15 \%$ require lipid-modifying therapy. ${ }^{37}$ For patients aged $>74$ years, who are not known to have CVD or to be at clinically determined high risk, "absolute risk over the next 5 years should be assessed using the Framingham Risk Equation Calculator using the age 74 years. Although this may underestimate the risk in this population, available evidence suggests that this approach will provide an estimate of minimum CVD risk". ${ }^{37}$

For those aged $>74$ years, "The decision to initiate therapy should be based on clinical judgment which takes into account the likely benefits and risks of treatment, life expectancy, co-morbidities and quality of life, personal values". 37

Table 5 lists the ESC/EAS recommendations for treatment of dyslipidemia in the elderly. ${ }^{35}$

Table 5 ESC/EAS recommendations for treatment of dyslipidemia in the elderly

\begin{tabular}{lll}
\hline Recommendations & $\begin{array}{l}\text { Class of } \\
\text { recommendation }\end{array}$ & $\begin{array}{l}\text { Level of } \\
\text { evidence }\end{array}$ \\
\hline $\begin{array}{l}\text { Treatment with statins is } \\
\text { recommended for elderly patients }\end{array}$ & B \\
with established CVD in the same & \\
way as for younger patients. & \\
Since elderly patients often have \\
comorbidities and have altered \\
pharmacokinetics, it is recommended to \\
start lipid-lowering medication at a low \\
dose and then titrate with caution to \\
achieve target lipid levels which are the \\
same as in the younger patients. \\
$\begin{array}{l}\text { Statin therapy may be considered } \\
\text { in elderly subjects free of CVD, }\end{array}$ \\
particularly in the presence of one \\
other CV risk factor besides age.
\end{tabular}

Note: Reprinted from The Lancet 2010;375. Jun M, Foote C, Lv J, et al. Effects of fibrates on cardiovascular outcomes: a systematic review and meta-analysis. 1875-1884. Copyright @2010, with permission from Elsevier. ${ }^{34}$ 


\section{National Institute \\ for Clinical Excellence (UK)}

The following statements were made in National Institute for Clinical Excellence (NICE) guidelines for lipid management in the elderly: "For the primary prevention of CVD in primary care, a systematic strategy should be used to identify people aged 40-74 who are likely to be at high risk"; "People aged 75 or older should be considered at increased risk of CVD, particularly people who smoke or have raised blood pressure. They are likely to benefit from statin treatment. Assessment and treatment should be guided by the benefits and risks of treatment, informed preference and comorbidities that may make treatment inappropriate". ${ }^{38}$

\section{International Atherosclerosis Society}

The recent IAS guidelines include a comprehensive section on lipid management in the elderly. ${ }^{17}$ Estimation of lifetime risk was recommended, in which it is assumed that $\mathrm{CV}$ risk burden remains constant beyond age 50 years. Because $\mathrm{CV}$ risk factors actually increase with age (Table 2), this may underestimate lifetime risk nevertheless. Calibration of estimates was recommended according to the country of residence, eg, CVD risk is lower in southern Europe and Asia compared with Northern Europe.

The IAS guidelines recommend estimating hard CHD risk in patients aged $>65$ years using Framingham scoring, recalibrated for country (http://hp2010.nhlbihin.net/aptiii/ calculator.asp?usertype=prof). Total CVD can be estimated by increasing the CHD risk by one-third, and the result should assist in deciding on statin therapy. ${ }^{17}$

\section{Other considerations}

Abelhafiz et al reviewed the relationship between cholesterol levels and outcomes in elderly patients especially with regard to body weight and frailty, suggesting that low body weight and frailty are important determinants of increased CVD and that these were associated with low cholesterol levels. ${ }^{39}$ While their discussion does not address the issue of statin therapy for CVD prevention, caution would appear appropriate in considering lipid treatment in frail and thin elderly patients. ${ }^{39}$

\section{Proposed guidelines for lipid management in the elderly}

All patients aged 65 years or more with one or more of the following CVD risk factors should be considered for treatment with lipid-modifying therapy:
1. Previous atherothrombotic cardiovascular disease (ATCVD).

2. Hypertension (BP $>150 / 80$ or on hypertensive therapy).

3. Low HDL-C $(<1.0 \mathrm{mmol} / \mathrm{L})$.

4. Diabetes mellitus.

5. Renal impairment (estimated glomerular filtration rate $<60 \mathrm{~mL} / \mathrm{min}$ ).

6. Metabolic syndrome (central obesity with one or more of low HDL-C $<1.0 \mathrm{mmol} / \mathrm{L}$, high TGs $>2.3 \mathrm{mmol} / \mathrm{L}$, fasting glucose $>6 \mathrm{mmol} / \mathrm{L}$ ).

7. Fasting TGs $>2.3 \mathrm{mmol} / \mathrm{L}$ (and high CVD risk).

8. Coronary calcium score $>300$ Agaston units.

9. Peripheral vascular disease (including demonstration of atherosclerotic plaques on arterial ultrasound).

10. Patients considered for statin therapy are required to have appropriate life expectancy for benefits of statin therapy to be realized. In most situations, a life expectancy of 5 years or more is reasonable.

11. Patients considered for statin therapy are required to have clear explanation of the rationale for statin therapy, the potential benefits and side effects, and the need for monitoring efficacy and tolerability.

12. Possible exceptions to the use of statins include the following:

a. Patients with low life expectancy $<5$ years

b. Patients with no recognized CVD risk factors in addition to one or more of the following:

i. Low coronary calcium score ( $<100$ Agaston units)

ii. Low LDL-C $(<2.5 \mathrm{mmol} / \mathrm{L})$ in the absence of secondary causes such as extreme weight loss, gastrointestinal disorders, or malignancy

c. Patients at increased risk of statin side effects

d. Low body mass (BMI $<18 \mathrm{~kg} / \mathrm{m}^{2}$ )

e. Women aged $>80$ years

f. Severe comorbidities

g. Previous ADRs with statins.

13. Statins should be used at minimal effective doses in order to achieve the required (target) LDL-C levels.

14. Target LDL-C levels are as follows:

a. At least 30\% reduction in LDL-C

b. And/or LDL-C $<2.5 \mathrm{mmol} / \mathrm{L}$ (patients without established CVD) and LDL-C $<1.8 \mathrm{mmol} / \mathrm{L}$ (patients with established CVD).

15. Patients on statins require more assiduous monitoring for adverse effects.

16. Ezetimibe can be used if statins are not tolerated, and can be added to low to moderate dose statin therapy in 
order to achieve desired target LDL-C levels. In this way, ADRs are likely to be minimized and LDL-C reduction is enhanced. This recommendation is consistent with the results of the IMProved Reduction of Outcomes: Vytorin Efficacy International Trial (IMPROVE IT) (to be presented late 2014). ${ }^{40}$

17. Fibrates are indicated to treat mild to moderate hypertriglyceridemia $(>2.3 \mathrm{mmol} / \mathrm{L})$ in the presence of low HDL-C $(<0.9 \mathrm{mmol} / \mathrm{L})$. Fibrates should be used for patients with $\mathrm{TG}>10 \mathrm{mmol} / \mathrm{L}$ to reduce the risk of pancreatitis as well as omega-3 fatty acids (EPA and DHA) 2-4 g/day.

18. Maintaining optimal nutrition is an important consideration in elderly patients, and the involvement of a dietitian in lipid management is recommended.

These recommendations are a consensus of the authors after reviewing the literature and current guidelines for treating lipids in the elderly population and in view of their clinical experience.

\section{Summary}

The decision to prescribe lipid-lowering therapy in the elderly for the prevention of CVD presents challenges. Clinical trial data demonstrates similar RRR as in middle-aged subjects and greater absolute risk reduction consistent with greater baseline absolute risk. In the most elderly ( $>80$ years), treatment decreases CVD events but not total mortality, consistent with high death rates from non-CVD illnesses. Our elderly warrant lipid-lowering therapy for primary and secondary prevention in the context of their absolute CV risk. The current therapeutic nihilism is not justified. It is time for clinicians to "Get with the Guidelines".

\section{Disclosure}

The authors report no conflicts of interest.

\section{References}

1. Simons LA, Simons J, Friedlander Y, McCallum J, Palaniappan L. Risk functions for prediction of cardiovascular disease in elderly Australians: the Dubbo Study. Med J Aust. 2003;178:113-116.

2. Golomb BA. Implications of statin adverse effects in the elderly. Expert Opin1 Drug Saf. 2005;4:389-397.

3. Hamilton-Craig I. Statin-associated myopathy. Med J Aust. 2001;175:486-489.

4. Yourman LC, Lee SJ, Schonberg MA, Widera EW, Smith AK. Prognostic indices for older adults: a systematic review. JAMA. 2012;307:182-192.

5. Massing MW, Foley KA, Sueta CA, et al. Trends in lipid management among patients with coronary artery disease: has diabetes received the attention it deserves? Diabetes Care. 2003;26:991-997.

6. Heeley EL, Peiris DP, Patel AA, et al. Cardiovascular risk perception and evidence - practice gaps in Australian general practice (the AusHEART study). Med J Aust. 2010;192:254-259.
7. Simons LA, Chung E. Are high coronary risk patients missing out on lipid-lowering drugs in Australia? Med J Aust. 2014;201: 213-216.

8. Hamilton-Craig I, Michealides C, Kostner K, Van Bockxmeer F. Current initiatives for detecting patients with familial hypercholesterolemia in primary care. Lipid Spin. 2013;11:5-10.

9. Holmes HM, Hayley DC, Alexander GC, Sachs GA. Reconsidering medication appropriateness for patients late in life. Arch Intern Med. 2006;166:605-609.

10. Australian Institute of Health and Welfare 2012. Australia's health 2012. Australia's health series no13. Cat no AUS 156. Canberra: AIHW. Available from http://www.aihw.gov.au/WorkArea/DownloadAsset. aspx?id=10737422169. Accessed 19 January, 2015.

11. Australian Institute of Health and Welfare 2008. Australia's health 2008. Cat no AUS 99. Canberra: AIHW. Available from http://www.aihw. gov.au/WorkArea/DownloadAsset.aspx?id=6442453674. Accessed 19 January, 2015.

12. Lloyd-Jones DM, Leip EP, Larson MG, et al. Prediction of lifetime risk for cardiovascular disease by risk factor burden at 50 years of age. Circulation. 2006;113:791-798.

13. Bang LM, Goa KL. Pravastatin: a review of its use in elderly patients. Drugs Aging. 2003;20:1061-1082.

14. Noaman S, Ibrahim JE, Grenfell R. Prescribing statins for cardiovascular disease prevention in the old: an absence of evidence and an absence of guidelines. Heart Lung Circ. 2014;23:619-624.

15. Treatment for cardiovascular disease. AIHW; 2013. Available from: http://www.aihw.gov.au/cardiovascular-health/treatment/. Accessed July 2, 2014.

16. Kannel WB. Coronary heart disease risk factors in the elderly. Am $J$ Geriatr Cardiol. 2002;11(2):101-107.

17. Prospective Studies Collaboration. Blood cholesterol and vascular mortality by age, sex, and blood pressure: a meta-analysis of individual data from 61 prospective studies with 55000 vascular deaths. Lancet. 2007;370:1829-1839.

18. International Atherosclerosis Society. An International Atherosclerosis Society Position Paper: Global Recommendations for the Management of Dyslipidemia; 2013. Available from: http://www.athero.org/download/ IASPPGuidelines_FullReport_2.pdf. Accessed July 2, 2014.

19. Hippisley-Cox J, Coupland C, Robson J, Brindle P. Derivation, validation, and evaluation of a new QRISK model to estimate lifetime risk of cardiovascular disease: cohort study using Q Research database. BMJ. 2010;341:c6624.

20. Berry JD, Dyer A, Cai X, et al. Lifetime risks of cardiovascular disease. N Engl J Med. 2012;366:321-329.

21. Jackson R, Kerr A, Wells S. Vascular risk calculators: essential but flawed clinical tools? Circulation. 2013;127:1929-1931.

22. Tota-Maharaj R, Blaha MJ, McEvoy JW, et al. Coronary artery calcium for the prediction of mortality in young adults $<45$ years old and elderly adults $>75$ years old. Eur Heart J. 2012;33:2955-2962.

23. Roberts CG, Guallar E, Rodriguez A. Efficacy and safety of statin monotherapy in older adults: a meta-analysis. J Gerontol A Biol Sci Med Sci. 2007;62:879-887.

24. Mihaylova B, Emberson J, Blackwell L, et al. The effects of lowering LDL cholesterol with statin therapy in people at low risk of vascular disease: meta-analysis of individual data from 27 randomised trials. Lancet. 2012;380:581-590.

25. Lloyd SM, Stott DJ, de Craen AJ, et al. Long-term effects of statin treatment in elderly people: extended follow-up of the PROspective Study of Pravastatin in the Elderly at Risk (PROSPER). PLoS One. 2013;8:e72642.

26. Allen Maycock CA, Muhlestein JB, Horne BD, et al. Statin therapy is associated with reduced mortality across all age groups of individuals with significant coronary disease, including very elderly patients. $J \mathrm{Am}$ Coll Cardiol. 2002;40:1777-1785.

27. Miettinen TA, Pyörälä K, Olsson AG, et al. Cholesterol-lowering therapy in women and elderly patients with myocardial infarction or angina pectoris. Circulation. 1997;96:4211-4218. 
28. Hunt D, Young P, Simes J, et al. Benefits of pravastatin on cardiovascular events and mortality in older patients with coronary heart disease are equal to or exceed those seen in younger patients: results from the LIPID trial. Ann Intern Med. 2001;134:931-940.

29. Lewis SJ, Moye LA, Sacks FM, et al. Effect of pravastatin on cardiovascular events in older patients with myocardial infarction and cholesterol levels in the average range. Results of the cholesterol and recurrent events (CARE) trial. Ann Intern Med. 1998;129: 681-689.

30. Al-Khadra S, Meisinger C, Amann U, et al. Secondary prevention medication after myocardial infarction: persistence in elderly people over the course of 1 year. Drugs Aging. 2014;31:513-525.

31. Sarwar N, Danesh J, Eiriksdottir G, et al. Triglycerides and the risk of coronary heart disease. Circulation. 2007;115:450-458.

32. Asia Pacific Cohort Studies Collaboration. Serum triglycerides as a risk factor for cardiovascular disease in the Asia-Pacific region. Circulation. 2004;110:2686-2687.

33. Kotseva K, Wood D, De Backer G, Pyorala K, Keil U, EUROASPIRE Study Group. EUROASPIRE III: A survey on the lifestyle, risk factors and use of cardioprotective drug therapies in coronary patients from 22 European countries. Eur J Cardiovasc Prev Rehabil. 2009; 16:121-137.

34. Jun M, Foote C, Lv J, et al. Effects of fibrates on cardiovascular outcomes: a systematic review and meta-analysis. Lancet. 2010;375: 1875-1884.

35. Catapano AL, Reiner Z, De Backer G, et al; ESC Committee for Practice Guidelines (CPG) 2008-2010 and 2010-2012 Committees, European Association for Cardiovascular Prevention and Rehabilitation. ESC/ EAS Guidelines for the management of dyslipidaemias. The Task Force for the management of dyslipidaemias of the European Society of Cardiology (ESC) and the European Atherosclerosis Society (EAS). Atherosclerosis. 2011;217:3-46.
36. Stone NJ, Robinson JG, Lichtenstein AH, et al. 2013 ACC/AHA guideline on the treatment of blood cholesterol to reduce atherosclerotic cardiovascular risk in adults: a report of the American College of Cardiology/American Heart Association Task Force on Practice Guidelines. J Am Coll Cardiol. 2014;63:2889-2934.

37. National Vascular Disease Prevention Alliance. Consensus statement for the prevention of vascular disease. Aust Fam Physician. 2004;33:235-239.

38. NICE clinical guideline. Cardiovascular risk assessment and the modification of blood lipids for the primary and secondary prevention of cardiovascular disease. NICE clinical guideline 67; 2010. Available from: http://www.nice.org.uk/guidance/cg67. Accessed July 2, 2014.

39. Abelhafiz AH, Loo BE, Hensey N, Bailey C, Sinclair A. The U-shaped relationship of traditional cardiovascular risk factors and adverse outcomes in later life. Aging Dis. 2012;3:454-464.

40. IMPROVE-IT: 'Modest' Benefit When Adding Ezetimibe to Statins in Post-ACS Patients. Available from: http:/www.medscape.com viewarticle/835030. Accessed 19 Jan 2015.
Vascular Health and Risk Management

\section{Publish your work in this journal}

Vascular Health and Risk Management is an international, peerreviewed journal of therapeutics and risk management, focusing on concise rapid reporting of clinical studies on the processes involved in the maintenance of vascular health; the monitoring, prevention and treatment of vascular disease and its sequelae; and the involvement of

\section{Dovepress}

metabolic disorders, particularly diabetes. This journal is indexed on PubMed Central and MedLine. The manuscript management system is completely online and includes a very quick and fair peer-review system, which is all easy to use. Visit http://www.dovepress.com/ testimonials.php to read real quotes from published authors. 